\title{
Closed loop GPR data inversion for soil hydraulic and electric property determination
}

\author{
S. Lambot, ${ }^{1,2}$ E. C. Slob, ${ }^{3}$ M. Vanclooster, ${ }^{2}$ and H. Vereecken ${ }^{1}$ \\ Received 17 August 2006; revised 5 October 2006; accepted 11 October 2006; published 10 November 2006.
}

[1] We propose an integrated electromagnetic-hydrodynamic inverse modeling approach for identifying field-scale unsaturated soil hydraulic properties and electric profiles from off-ground time-lapse ground-penetrating radar data. Hydrodynamic modeling based on the one-dimensional Richards' equation with homogeneous soil hydraulic parameters is used to physically constrain the full-wave radar electromagnetic inverse problem in a closed loop. Significant effects can be observed of water dynamics on the time-lapse radar data and numerical studies demonstrate the uniqueness of the inverse solution. The results suggest that the method is promising for non-invasive characterization of the shallow subsurface and monitoring of its dynamics. Citation: Lambot, S., E. C. Slob, M. Vanclooster, and H. Vereecken (2006), Closed loop GPR data inversion for soil hydraulic and electric property determination, Geophys. Res. Lett., 33, L21405, doi:10.1029/2006GL027906.

\section{Introduction}

[2] Sustainable agricultural and environmental management of water and land resources particularly relies on the description and understanding of near-surface water distribution and dynamics. Obtaining this information with the required spatio-temporal resolution is however complicated by the inaccessibility of the subsurface and the inherent variability of the soil properties. As the dielectric permittivity of liquid water overwhelms the permittivity of other soil components, water principally governs electromagnetic wave propagation in the soil and permits therefore the use of high resolution and non-invasive geophysical techniques to indirectly measure its quantity. In that respect, groundpenetrating radar (GPR) has proven to be successful in many hydrological applications. Reviews are given by Huisman et al. [2003] and Annan [2005].

[3] In particular, time-lapse measurements permit to monitor subsurface flow processes [Tsoflias et al., 2001], which provides valuable information on the soil hydraulic properties when coupled with hydrodynamic modeling. Recently, Binley et al. [2002], Rucker and Ferré [2004], Kowalsky et al. [2005], and Cassiani and Binley [2005] used cross-borehole transmission GPR and travel time tomographic inversion techniques to monitor the distribution of water between boreholes and infer soil hydraulic

\footnotetext{
${ }^{1}$ Agrosphere (ICG IV), Institute of Chemistry and Dynamics of the Geosphere, Forschungszentrum Jülich GmbH, Jülich, Germany.

${ }^{2}$ Department of Environmental Sciences and Land Use Planning, Université catholique de Louvain, Louvain-la-Neuve, Belgium.

${ }^{3}$ Department of Geotechnology, Delft University of Technology, Delft, Netherlands.
}

Copyright 2006 by the American Geophysical Union. 0094-8276/06/2006GL027906\$05.00 properties. Lambot et al. [2004a] used far-field reflection GPR and full-wave electromagnetic inversion to monitor infiltration in a soil column and subsequently applied hydrodynamic inversion to identify the hydraulic conductivity and water retention functions of the soil.

[4] While borehole GPR is essentially useful for characterizing locally the subsurface to substantial depths, surface and off-ground GPR techniques are needed to cover large areas with a high spatial resolution. This is particularly relevant for applications in surface hydrology or agriculture, where the shallow vadose zone is of primary interest [Lunt et al., 2005]. A commonly used method to retrieve vertical dielectric profiles is the common midpoint (CMP) method [Greaves et al., 1996]. This approach requires surface multioffset radar soundings at a fixed central location to extract stacking velocity fields, generally using the straightray approximation. This also requires the velocity to be homogeneous laterally below the whole CMP aperture. For off-ground mode, which is the most practical configuration for real-time mapping, Lambot et al. [2004c] recently proposed a method based on full-wave inversion of monostatic ultra-wide band frequency domain radar data, thereby maximizing information retrieval from a single GPR measurement. Both phase (travel time) and amplitude information are inherently exploited. The technique relies on an accurate and computationally effective radar forward model, which includes internal antenna and antenna-soil interaction propagation effects and solves exactly the three-dimensional (3-D) Maxwell's equations for wave propagation in multilayered media. For this method only within the first Fresnel zone we expect the lateral change in velocity to be negligible, plus the waves we measure travel mostly vertically down and up and most other directions are not recorded.

[5] Whether for borehole or surface and off-ground GPR, a major concern to any reconstruction approach is the uniqueness of the inverse solution [Spagnolini, 1997; Zhou et al., 2001; Ghose and Slob, 2006], which depicts in addition to parameter identifiability the physical frontiers of information retrieval from the available data. This is particularly limiting when facing inhomogeneous and continuously variable dielectric media [Hashish, 2003; Lambot et al., 2004b], as prevalent in the environment.

[6] In this paper, we propose a new integrated inverse modeling approach for identifying in a non-invasive way near-surface hydraulic properties and electric profiles at the field-scale from time-lapse off-ground GPR data. Electromagnetic inversion of the radar data is physically constrained in a closed loop using hydrodynamic modeling, thereby reducing the solution space to solutions honoring soil hydrodynamic laws. A synthetic infiltration experiment in a sandy soil is performed to demonstrate the theoretical applicability of the method, i.e., that enough information 


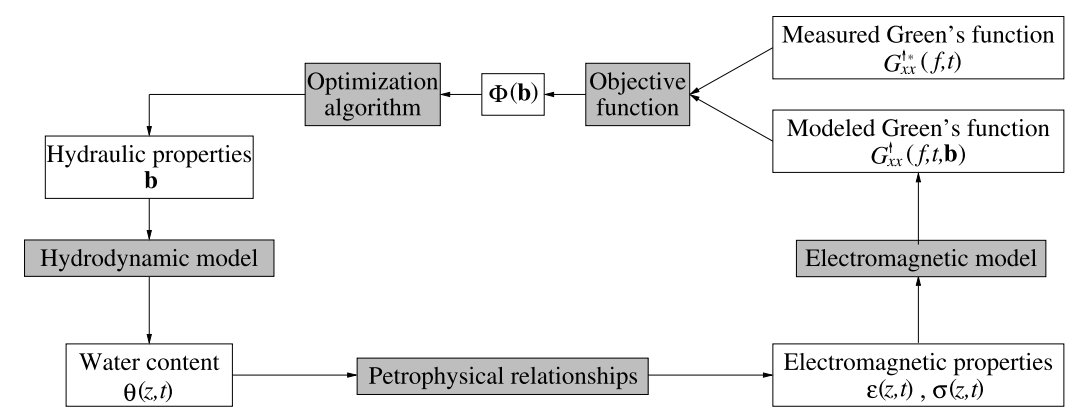

Figure 1. Flowchart representing the integrated electromagnetic and hydrodynamic inversion of time-lapse radar measurements for estimating soil hydraulic properties and electric profiles ( $t$ is time, $z$ is depth, and $f$ is frequency). Shaded boxes denote operators and white boxes denote variables.

may be contained in the time-lapse radar data to identify the soil hydrogeophysical properties.

\section{Closed Loop Inversion}

[7] The proposed closed loop electromagnetic-hydrodynamic inversion procedure is depicted in Figure 1. It particularly applies to ultra-wide band continuous-wave stepped-frequency off-ground monostatic GPR, for which accurate full-wave signal forward modeling is available [Lambot et al., 2004c]. To reduce model non-uniqueness and optimization issues in retrieving vertical permittivity profiles, we choose the profile that satisfies the soil hydrodynamic laws. This is achieved by incorporating a hydrodynamic model as a constraint in the inversion process. Hence, the solution space reduces and inherent uniqueness issues may be partly or completely resolved. In addition, it provides the soil hydraulic properties. This procedure is especially advantageous as the required hydrodynamic boundary conditions can be intrinsically obtained in a non-invasive way. The remaining unknowns are the initial conditions in the hydrodynamic model, soil hydraulic properties, and soil-specific petrophysical relationships that correlate water content $(\theta)$ to dielectric permittivity $(\varepsilon)$ and electric conductivity $(\sigma)$. In this paper, we assume that only the soil hydraulic properties are unknown, which define the parameter vector $\mathbf{b}$ to be estimated. Moreover, we assume water flow to be 1-D in the vertical $z$-direction with homogeneous hydraulic properties.

[8] The measured and modeled time-lapse GPR data are represented by the full-wave GPR Green's functions $G_{x x}^{\uparrow *}(f, t)$ and $G_{x x}^{\uparrow}(f, t, \mathbf{b})$, respectively, defined for wave propagation in multilayered media [Michalski and Mosig, 1997; Slob and Fokkema, 2002], where $f$ is the radar operating frequency and $t$ is the time variable for the hydrodynamic event. Given the monostatic mode of operation, the sensitivity of the radar measurements with respect to horizontal variations in the soil electromagnetic parameters is expected to be negligible. Continuous variations of these quantities with depth can be emulated by considering layer thicknesses sufficiently small compared to the minimal wavelength $\lambda$, typically $\lambda / 10$. The Green's function can be obtained from the raw radar data by filtering all antenna effects and represents the $x$-directed (first subscript $x$ in $G_{x x}^{\uparrow}$ ) component of the backscattered (up arrow in $G_{x x}^{\uparrow}$ ) electric field at the antenna phase center for a unit-strength and $x$-directed (second subscript $x$ in $G_{x x}^{\uparrow}$ ) electric source, also situated at the antenna phase center [Lambot et al., 2004c].

[9] The inverse problem is formulated by the leastsquares criterion in terms of electromagnetic data and the objective function to minimize is accordingly defined as follows:

$$
\phi(\mathbf{b})=\sum_{t} \sum_{f}\left|G_{x x}^{\uparrow *}(f, t)-G_{x x}^{\uparrow}(f, t, \mathbf{b})\right|^{2}
$$

[10] Since the Green's function is a complex quantity, the difference between observed and modeled data is expressed by the amplitude of the differences in the complex plane. Equation (1) relates indirectly the time-lapse response of the porous medium to its constitutive hydraulic parameters. However, as in most electromagnetic inverse problems, this function is nonlinear and may be characterized by multiple local minima. Such a topography necessitates the use of a robust global optimization algorithm. We use the global multilevel coordinate search [Huyer and Neumaier, 1999] that we combine sequentially with the classical Nelder-Mead simplex [Lagarias et al., 1998].

\section{Numerical Experiment}

[11] A synthetic experiment has been performed to investigate the well-posedness of the inverse problem defined above. We simulated a transient infiltration event in a sandy soil, for which actual hydraulic properties and petrophysical relationships were simultaneously available and for which the adequacy of the hydrodynamic model described below has been demonstrated [Lambot et al., 2002, 2004c].

[12] For fluid flow modeling, we implemented the numerical solution of WAVE [Vanclooster et al., 1996], where the differential Richards' equation is approximated by finite differences with an implicit discretization scheme and with an explicit linearization of the hydraulic conductivity and differential moisture capacity. The soil hydraulic properties were described by the non-hysteretic unimodal Mualem-van Genuchten model [Mualem, 1976; van Genuchten, 1980], resulting in 6 hydraulic parameters. Their values assumed for the sand are $\theta_{r}=0$ (residual water content), $\theta_{s}=0.357$ (saturated water content), $\alpha=0.0278 \mathrm{~cm}^{-1}$ (air entry value parameter), $n=7.02$ (pore size distribution parameter), $K_{s}=8.24 \mathrm{~cm} \mathrm{~min}^{-1}$ (saturated hydraulic conductivity), 


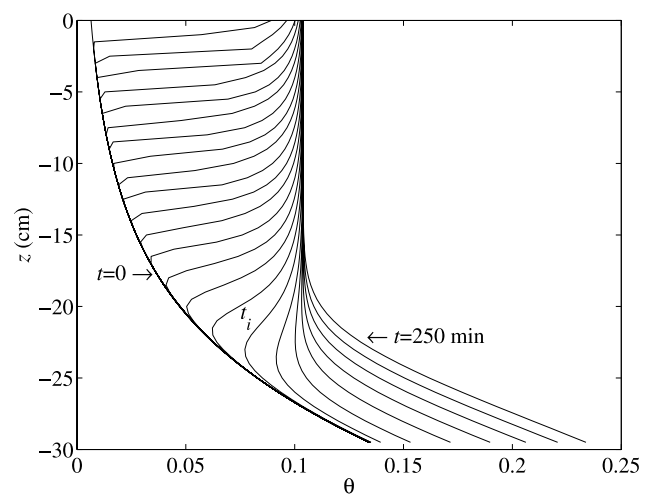

Figure 2. Volumetric water content $(\theta)$ as a function of depth $(z)$ and time of infiltration $(t)$ in the sandy soil.

and $\lambda=2.89$ (tortuosity). In the inversion process, we assume $\theta_{r}$ and $\theta_{s}$ to be known, as these parameters are readily accessible (e.g., they may be obtained from the radar data in dry and saturated conditions, respectively). The resulting parameter vector to be estimated is then defined as $\mathbf{b}=\left[\alpha, n, K_{s}, \lambda\right]$. The spatial flow domain was discretized into 60 equidistant linear elements, representing each $0.5 \mathrm{~cm}$ of the $30 \mathrm{~cm}$ length domain. The top boundary condition was a constant downward flux $\left(q=0.01 \mathrm{~cm} \mathrm{~min}^{-1}\right)$ and the bottom condition was chosen as a seepage face. The initial pressure head condition was a hydrostatic equilibrium with a water table at $70 \mathrm{~cm}$ depth. Figure 2 represents the simulated water content with respect to time and depth. We used the models of Rhoades et al. [1976] and Ledieu et al. [1986] to relate water content to dielectric permittivity and electric conductivity.

[13] Figure 3 shows the synthetic Green's function for the simulated infiltration, with respect to radar frequency and infiltration time. This constitutes radar observations. We used an evenly spaced subset of 25 infiltration times and 91 frequencies for the inversion, as it already captures major information from the infiltration event. The electromagnetic wave propagation domain has been discretized into 60 homogeneous layers, emulating the continuity of the electric profiles. We can clearly observe the significant effect of moisture variations with time on $G_{x x}^{\uparrow}$ (see also Figure 2), which lead to some oscillations, depending on frequency. After about $200 \mathrm{~min}$, the effect of water dynamics, occurring mainly below $20 \mathrm{~cm}$ depth, becomes negligible in the whole frequency range. An analysis of the radar data in the time domain shows that only the surface reflection is still visible (see Figure 3c). We demonstrated that this does not originate from electric losses, but from the particular shape of the continuous dielectric profile, which precludes radar reflections from the underground [Hashish, 2003]. In that respect, it is worth noting that the water table is also not visible before the infiltration event.

\section{Results}

[14] Figure 4 represents cross sections of the 4-D objective function with respect to all parameter pairs, while the other parameters are held constant at their true value. The white areas represent parameter sets for which the numerical hydrodynamic model does not converge. They usually correspond to unrealistic parameter combinations, but also to numerical limitations. First, we can observe in each cross section a unique global minimum. Parameters $\alpha$ and $n$ are negatively correlated, with a poor sensitivity for $n$, leastwise for large values. $K_{s}$ is correlated positively with the other parameters, but is well defined. It is worth noting that the tortuosity $\lambda$, which is usually disregarded in soil hydrodynamics (usually assumed to be equal to 0.5), is quite sensitive.

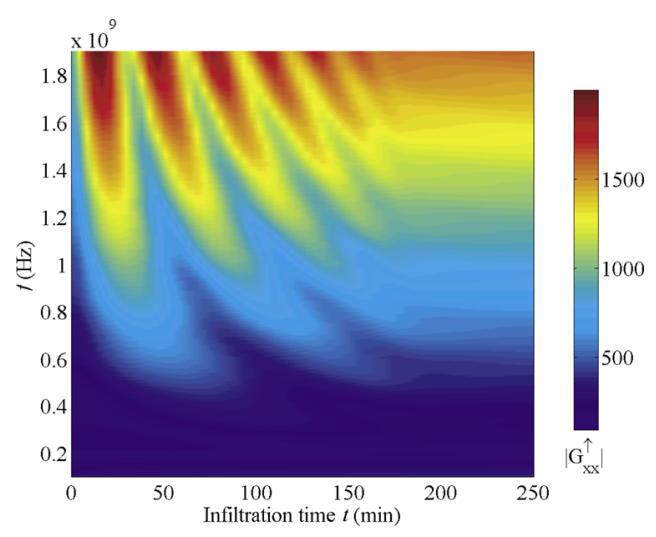

(a)

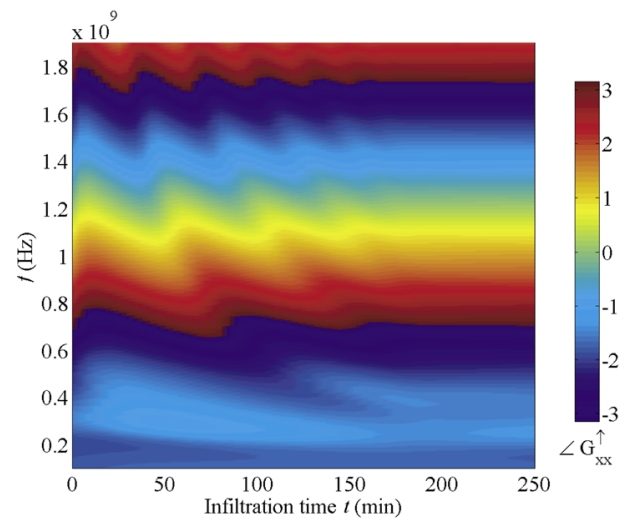

(b)

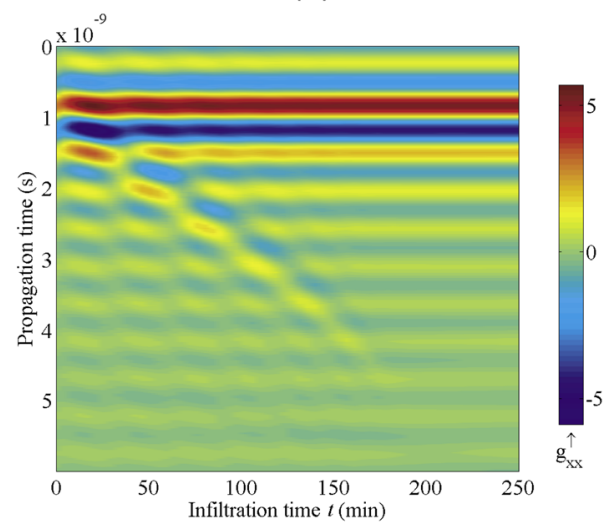

(c)

Figure 3. (a) Amplitude and (b) phase of the synthetic frequency domain GPR Green's function $\left(G_{x x}^{\uparrow *}\right)$ with respect to the infiltration time (t), $f$ being the frequency. (c) Time domain representation of the Green's function. 


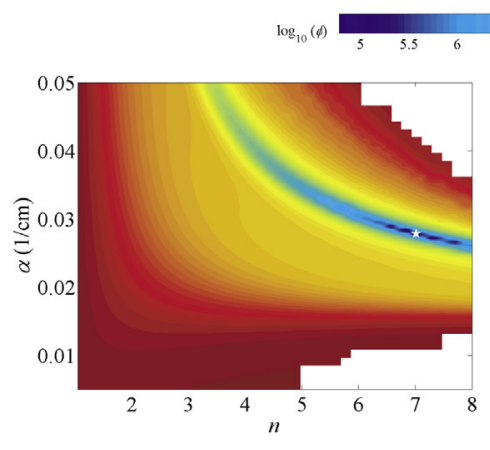

(a)

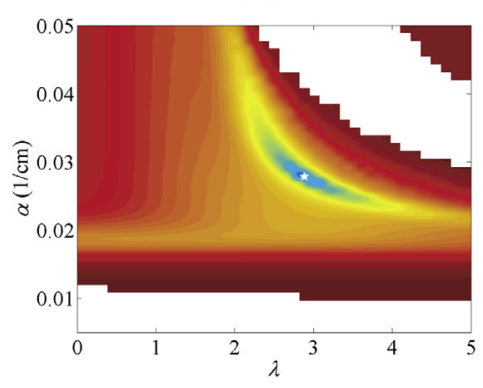

(c)

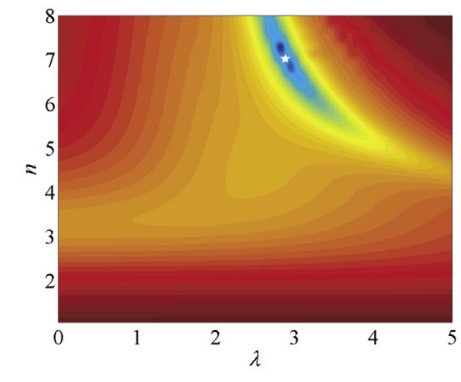

(e)

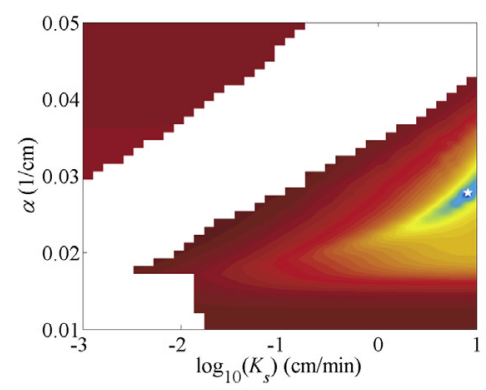

(b)

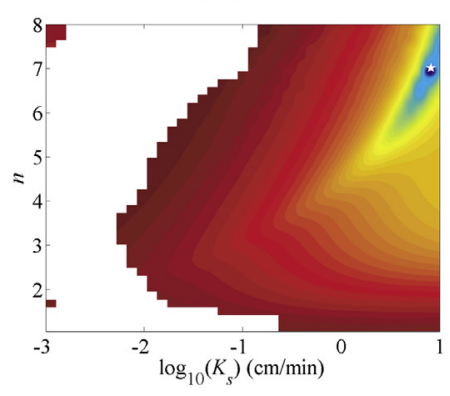

(d)

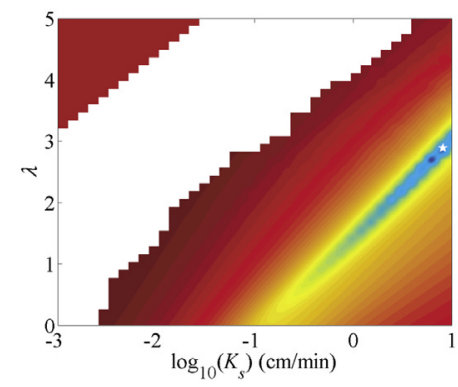

(f)

Figure 4. Response surfaces of the objective function logarithm $\log _{10}(\phi)$ in the (a) $\alpha-n$, (b) $\alpha-K_{s}$, (c) $\alpha-\lambda$, (d) $n-K_{s}$, (e) $n-\lambda$, and (f) $\lambda-K_{s}$ parameter planes. The white pentagram marker represents the true parameter values, which correspond exactly to the global minimum of the objective function. The white areas correspond to parameter sets for which the hydrodynamic model does not converge.

[15] If topography analysis provides valuable information on parameter correlation, sensitivity and for designing an efficient optimization strategy, these 2-D views of $\phi(\mathbf{b})$ does not demonstrate uniqueness and calculating $\phi(\mathbf{b})$ in the whole parameter space would demand tremendous computation resources. Therefore, we used the above mentioned optimization procedure to minimize $\phi(\mathbf{b})$ and test if the found solution corresponds to the true parameter set. In spite of the large parameter space considered (see Figure 4), covering major soils of the world as far as Mualem-van Genuchten's model is concerned, we observed that the obtained results correspond exactly to the true parameter values. The solution is reached after about 800 iterations. The computation time is about 7 hours using 16 processors in parallel on the JUMP cluster from the Forschungszentrum Jülich. This shows the necessity of regularizing the GPR data inversion. It is worth noting that in this example the position of the global minimum in the parameter space, quite close to the bounding box, does not especially advantage the global optimization procedure [see Lambot et al., 2002].

\section{Conclusions}

[16] We have demonstrated that enough information may be contained in the time-lapse GPR measurements when constrained with fluid flow modeling to identify the soil hydraulic properties and retrieve shallow soil electric or moisture profiles. The remaining issues to investigate are the stability of the inverse solution with respect to actual electromagnetic and hydrodynamic modeling errors (e.g., uncertain initial and boundary conditions), to measurement errors, and to errors on the petrophysical relationships. In addition, the uniqueness and optimization questions when additional unknowns are considered in the inversion procedure (e.g., when hydraulic properties vary with depth) should be also addressed. Yet, the proposed technique appears to be promising for mapping the shallow subsurface 
(e.g., root zone) hydraulic properties at the field scale using GPR and potentially at larger scales using airborne and spaceborne ultra-wide band radar remote sensing.

[17] Acknowledgments. This research was supported by a Marie Curie Intra-European Fellowship within the 6th European Community Framework Programme, Delft University of Technology (Netherlands), the Université catholique de Louvain (Belgium), and the Forschungszentrum Jülich GmbH (Germany). The authors are grateful to Oliver Bücker for implementing the codes on the JUMP cluster of the Forschungszentrum Jülich.

\section{References}

Annan, A. P. (2005), GPR methods for hydrogeological studies, in Hydrogeophysics, edited by Y. Rubin and S. Hubbard, p. 532, Springer, New York.

Binley, A., G. Cassiani, R. Middleton, and P. Winship (2002), Vadose zone flow model parameterisation using cross-borehole radar and resistivity imaging, J. Hydrol., 267, 147-159.

Cassiani, G., and A. Binley (2005), Modeling unsaturated flow in a layered formation under quasi-steady state conditions using geophysical data constraints, Adv. Water Resour., 28, 467-477.

Ghose, R., and E. C. Slob (2006), Quantitative integration of seismic and GPR reflections to derive unique estimates for water saturation and porosity in subsoil, Geophys. Res. Lett., 33, L05404, doi:10.1029/ 2005GL025376.

Greaves, R. J., D. P. Lesmes, J. M. Lee, and M. N. Toksov (1996), Velocity variations and water content estimated from multi-offset, ground-penetrating radar, Geophysics, 61, 683-695.

Hashish, E. A. (2003), Forward and inverse scattering from an inhomogeneous dielectric slab, J. Electromagn. Waves Appl., 17, 719-736.

Huisman, J. A., S. S. Hubbard, J. D. Redman, and A. P. Annan (2003), Measuring soil water content with ground penetrating radar: A review, Vadose Zone J., 2, 476-491.

Huyer, W., and A. Neumaier (1999), Global optimization by multilevel coordinate search, J. Global Optim., 14, 331-355.

Kowalsky, M. B., S. Finsterle, J. Peterson, S. Hubbard, Y. Rubin, E. Majer, A. Ward, and G. Gee (2005), Estimation of field-scale soil hydraulic and dielectric parameters through joint inversion of GPR and hydrological data, Water Resour. Res., 41, W11425, doi:10.1029/2005WR004237.

Lagarias, J. C., J. A. Reeds, M. H. Wright, and P. E. Wright (1998), Convergence properties of the Nelder-Mead simplex method in low dimensions, SIAM J. Optim., 9, 112-147.

Lambot, S., M. Javaux, F. Hupet, and M. Vanclooster (2002), A global multilevel coordinate search procedure for estimating the unsaturated soil hydraulic properties, Water Resour. Res., 38(11), 1224, doi:10.1029/ 2001WR001224.

Lambot, S., M. Antoine, I. van den Bosch, E. C. Slob, and M. Vanclooster (2004a), Electromagnetic inversion of GPR signals and subsequent hydrodynamic inversion to estimate effective vadose zone hydraulic properties, Vadose Zone J., 3, 1072-1081.
Lambot, S., J. Rhebergen, I. van den Bosch, E. C. Slob, and M. Vanclooster (2004b), Measuring the soil water content profile of a sandy soil with an off-ground monostatic ground penetrating radar, Vadose Zone J., 3, $1063-1071$.

Lambot, S., E. C. Slob, I. van den Bosch, B. Stockbroeckx, and M. Vanclooster (2004c), Modeling of ground-penetrating radar for accurate characterization of subsurface electric properties, IEEE Trans. Geosci. Remote Sens., 42, $2555-2568$

Ledieu, J., P. D. Ridder, P. D. Clercq, and S. Dautrebande (1986), A method of measuring soil moisture by time domain reflectometry, J. Hydrol., 88 , $319-328$

Lunt, I. A., S. S. Hubbard, and Y. Rubin (2005), Soil moisture content estimation using ground-penetrating radar reflection data, J. Hydrol., 307, 254-269.

Michalski, K. A., and J. R. Mosig (1997), Multilayered media Green's functions in integral equation formulations, IEEE Trans. Antennas Propag., 45, 508-519.

Mualem, Y. (1976), A new model for predicting the hydraulic conductivity of unsaturated porous media, Water Resour. Res., 12, 513-522.

Rhoades, J. D., P. A. C. Raats, and R. J. Prather (1976), Effects of liquidphase electrical conductivity, water content, and surface conductivity on bulk soil electrical conductivity, Soil Sci. Soc. Am. J., 40, 651-655.

Rucker, D. F., and T. P. A. Ferré (2004), Parameter estimation for soil hydraulic properties using zero-offset borehole radar: Analytical method, Soil Sci. Soc. Am. J., 68, 1560-1567.

Slob, E. C., and J. Fokkema (2002), Coupling effects of two electric dipoles on an interface, Radio Sci., 37(5), 1073, doi:10.1029/2001RS002529.

Spagnolini, U. (1997), Permittivity measurements of multilayered media with monostatic pulse radar, IEEE Trans. Geosci. Remote Sens., 35, 454-463.

Tsoflias, G. P., T. Halihan, and J. M. Sharp (2001), Monitoring pumping test response in a fractured aquifer using ground-penetrating radar, Water Resour. Res., 37, 1221-1229.

Vanclooster, M., P. Viaene, J. Diels, and K. Christiaens (1996), WAVE, a mathematical model for simulating water and agrochemicals in the soil and vadose environment, release 2.1, report, Inst. for Land and Water Manage., Univ. catholique de Louvain, Louvain-la-Neuve, Belgium.

van Genuchten, M. T. (1980), A closed-form equation for predicting the hydraulic conductivity of unsaturated soils, Soil Sci. Soc. Am. J., 44, $892-898$

Zhou, C., L. Liu, and J. W. Lane (2001), Nonlinear inversion of boreholeradar tomography data to reconstruct velocity and attenuation distribution in earth materials, J. Appl. Geophys., 47, 271-284.

S. Lambot and H. Vereecken, Agrosphere (ICG IV), Institute of Chemistry and Dynamics of the Geosphere, Forschungszentrum Jülich GmbH, D-52425 Jülich, Germany. (s.lambot@fz-juelich.de)

E. C. Slob, Department of Geotechnology, Delft University of Technology, Mijnbouwstraat 120, NL-2628 RX Delft, Netherlands.

M. Vanclooster, Department of Environmental Sciences and Land Use Planning, Université catholique de Louvain, Croix du Sud 2 Box 2, B-1348 Louvain-la-Neuve, Belgium. 